International Journal of Pure and Applied Mathematics

Volume 115 No. 3 2017, 477-496

ISSN: 1311-8080 (printed version); ISSN: 1314-3395 (on-line version)

url: http://www.ijpam.eu

doi: 10.12732 /ijpam.v115i3.3

ijpam.eu

\title{
COMMON FIXED POINT AND BEST PROXIMITY POINT THEOREMS IN C*-ALGEBRA-VALUED METRIC SPACES
}

\author{
Saranan Mondal ${ }^{1}$, Ankush Chanda ${ }^{2}$, Surajit Karmakar ${ }^{3}$ \\ 1,2,3 Department of Mathematics \\ National Institute of Technology \\ Durgapur, West Bengal, INDIA
}

\begin{abstract}
In the article, we prove the existence and uniqueness of common fixed points for self-maps with contractive or expansive conditions on $C^{*}$-algebra-valued metric spaces without taking the continuity assumption on either of the mappings $S$ or $T$. Also, we define $C^{*}$-algebravalued proximal contraction and show the existence and uniqueness of best proximity points for these proximal contraction mappings on the said spaces. Moreover, the paper provides an application to prove the existence and uniqueness of the solution for a type of integral equations.
\end{abstract}

AMS Subject Classification: $C^{*}$-algebra-valued metric space, expansion mapping, $C^{*}$ algebra-valued proximal contraction, common fixed point, best proximity point

Key Words: 47H10, 54H 25

\section{Introduction}

Indeed, $C^{*}$-algebras are important areas of research in functional analysis [[3], [7]-[8],[10]-[11]]. It is generally believed that $C^{*}$-algebras were first considered primarily for their use in quantum mechanics to model algebras of physical observables. Although it has a great impact in operator theory on Hilbert spaces. The term $C^{*}$-algebra was introduced by I. Segal [10] in 1947 to describe normclosed subalgebras of $B(H)$, namely, the space of all bounded operators on Hilbert space $H$. In his paper [10], Segal defined a $C^{*}$-algebra as a "uniformly closed, self-adjoint algebra of bounded operators on a Hilbert space". Very re-

$\begin{array}{lr}\text { Received: } & \text { December 14, } 2016 \\ \text { Revised: } & \text { March 23, } 2017 \\ \text { Published: } & \text { July 27, } 2017\end{array}$

(c) 2017 Academic Publications, Ltd. url: www.acadpubl.eu

${ }^{\S}$ Correspondence author 
cently, Ma et al. [7] introduced the notion of $C^{*}$-algebra-valued metric spaces and proved some fixed point theorems for self-maps with contractive or expansive conditions on such spaces. Banking on these concepts, our paper deals with some common fixed point theorems and best proximity theorem on the said spaces and also offers a scope of application of the theory for a type of integral equations.

We get into the paper with the preliminary ideas of $C^{*}$-algebras. Let $\mathbb{A}$ be a unital algebra with the identity element $I$. An involution on $\mathbb{A}$ is a conjugate linear map $a \mapsto a^{*}$ on $\mathbb{A}$ with $a^{* *}=a$ and $(a b)^{*}=b^{*} a^{*}$ for all $a, b \in \mathbb{A}$. The pair $(\mathbb{A}, *)$ is called a $*$-algebra. A $*$-algebra with complete sub-multiplicative norm such that $\left\|a^{*}\right\|=\|a\|$ for all $a \in \mathbb{A}$ is known as a Banach $*$-algebra. Moreover, a $C^{*}$-algebra is a Banach $*$-algebra with $\left\|a^{*} a\right\|=\|a\|^{2}$.

Moreover, there exist a lot of rich works involving fixed points using the Banach contraction principle [1]. Subsequently this result has been extended and generalized in different directions such as common fixed point theorems, coupled fixed point theorems and at the same time other extensions like best proximity theorems by various authors [see [2]-[7] and references therein].

In this paper, we obtain common fixed points for two self-mappings satisfying contractive type or expansive type conditions in a $C^{*}$-algebra-valued metric space. Our results generalize and improve the results in [7]. Moreover, we introduce new concepts of proximal contraction mappings in this structure and establish best proximity point theorems for such type of mappings there.

This paper unfolds with some definitions and notations that have been utilized in our subsequent discussions and these are noted down in the technical preliminaries chapter. In Section 3, we prove some common fixed point theorems for two self-mappings for contractive or expansion mapping in $C^{*}$-algebravalued metric spaces without taking the continuity assumption on either of the mappings $S$ or $T$. Moreover in Section 4, we define $C^{*}$-algebra-valued proximal contraction in the said spaces and consequently we prove a best proximity point theorem for these proximal contractions. Finally in the last section we illustrate an application of the aforesaid theorems for a type of integral equations.

\section{Technical Preliminaries}

To begin with, let us put down some basic definitions and notations that have served our purpose throughout the paper.

We consider $\mathbb{A}$ as an unital $C^{*}$-algebra with unit $I$ and set

$$
\mathbb{A}_{h}=\left\{x \in \mathbb{A}: x=x^{*}\right\}
$$


An element $x \in \mathbb{A}$ is called positive if $x \in \mathbb{A}_{h}$ and $\sigma(x) \subset \mathbb{R}_{+}=[0, \infty)$, where $\sigma(x)$ is the spectrum of $x$ and it is denoted by $x \succeq \theta$. Also we consider

$$
\mathbb{A}_{+}=\{x \in \mathbb{A}: x \succeq \theta\} \text { and }\|x\|=\left(x^{*} x\right)^{\frac{1}{2}} .
$$

Remark 2.1. [11] When $\mathbb{A}$ is a unital $C^{*}$-algebra, then for any $x \in \mathbb{A}_{+}$, we have, $x \preceq I \Leftrightarrow\|x\| \leq 1$.

Definition 2.2. [7] Let $X$ be a non-empty set. Suppose the mapping $d: X \times X \rightarrow \mathbb{A}$ satisfies:

(1) $\theta \preceq d(x, y)$ for all $x, y \in X$ and $d(x, y)=\theta \Leftrightarrow x=y$;

(2) $d(x, y)=d(y, x)$ for all $x, y \in X$;

(3) $d(x, y) \preceq d(x, z)+d(z, y)$ for all $x, y, z \in X$.

Then $d$ is called a $C^{*}$-algebra-valued metric on $X$ and $(X, \mathbb{A}, d)$ is called a $C^{*}$-algebra-valued metric space.

Definition 2.3. [7] Let $(X, \mathbb{A}, d)$ be a $C^{*}$-algebra-valued metric space. Suppose that $\left\{x_{n}\right\} \subset X$ and $x \in X$. If for any $\varepsilon>0$, there exists $N$ such that for all $n>N,\left\|d\left(x_{n}, x\right)\right\| \leq \varepsilon$, then $\left\{x_{n}\right\}$ is said to be convergent with respect to $\mathbb{A}$ and $\left\{x_{n}\right\}$ converges to $x$ and we say $x$ is the limit of $\left\{x_{n}\right\}$. We denote it by $\lim _{n \rightarrow \infty} x_{n}=x$.

Definition 2.4. [7] Let $(X, \mathbb{A}, d)$ be a $C^{*}$-algebra-valued metric space. Suppose that $\left\{x_{n}\right\} \subset X$ and $x \in X$. If for any $\varepsilon>0$, there exists $N$ such that for all $n, m>N,\left\|d\left(x_{n}, x_{m}\right)\right\| \leq \varepsilon$, then $\left\{x_{n}\right\}$ is said to be a Cauchy sequence with respect to $\mathbb{A}$.

We say $(X, \mathbb{A}, d)$ is a complete $C^{*}$-algebra-valued metric space if every Cauchy sequence with respect to $\mathbb{A}$ is convergent.

It is clear that if $X$ is a Banach space with $d(x, y)=\|x-y\| I$, then $(X, \mathbb{A}, d)$ is a complete $C^{*}$-algebra-valued metric space. There are some nontrivial examples of complete $C^{*}$-algebra-valued metric spaces. For detailed study one is referred to see Examples (2.1 \& 2.2) of [7].

Definition 2.5. (cf. [7]) Suppose $(X, \mathbb{A}, d)$ is $C^{*}$-algebra-valued metric space. We say a mapping $T: X \rightarrow X$, is a $C^{*}$-algebra-valued contractive mapping on $X$ if there exists a $P \in \mathbb{A}$ with $\|P\|<1$ such that

$$
d(T x, T y) \preceq P^{*} d(x, y) P, \quad \forall x, y \in X
$$




\section{Common Fixed Point Theorem}

In this section, we present the existence and uniqueness of common fixed points of two self-mappings with contractive or expansive conditions on $C^{*}$-algebravalued metric spaces and also their proofs.

To prove the existence and uniqueness of common fixed points, the following lemmas are necessary.

Lemma 3.1. Let a sequence $\left\{x_{n}\right\}$ in $C^{*}$-algebra-valued metric space $(X, \mathbb{A}, d)$ and $h \in[0,1)$. If $\lambda_{n}=\left\|d\left(x_{n+1}, x_{n}\right)\right\|$ satisfies

$$
\lambda_{n} \leq h \lambda_{n-1},
$$

then $\left\{x_{n}\right\}$ is a Cauchy sequence.

Proof. Let us consider $\lambda_{n}=\left\|d\left(x_{n+1}, x_{n}\right)\right\|$.

Thus for $h=[0,1)$,

$$
\lambda_{n} \leq h \lambda_{n-1} \leq h^{2} \lambda_{n-2} \leq \cdots \leq h^{n} \lambda_{0} \text { for all } n \in \mathbb{N} .
$$

Let $m, n \in \mathbb{N}$ such that $m>n \geq n_{1}$. Then

$$
\begin{aligned}
\left\|d\left(x_{m}, x_{n}\right)\right\| & \leq\left\|d\left(x_{m}, x_{m-1}\right)\right\|+\left\|d\left(x_{m-1}, x_{m-2}\right)\right\|+\cdots+\left\|d\left(x_{n+1}, x_{n}\right)\right\| \\
& =\lambda_{m-1}+\lambda_{m-2}+\cdots+\lambda_{n} \\
& \leq\left(h^{m-1}+h^{m-2}+\cdots+h^{n}\right) \lambda_{0} \\
& =h^{n}\left(1+h+h^{2}+h^{3}+\cdots+h^{m-n-1}\right) \lambda_{0} \\
& \leq \frac{h^{n}}{1-h} \lambda_{0} \rightarrow 0
\end{aligned}
$$

as $n \rightarrow \infty$. Therefore $\left\{x_{n}\right\}$ is a Cauchy sequence in $X$ with respect to $\mathbb{A}$.

Lemma 3.2. Let a sequence $\left\{x_{n}\right\}$ in $C^{*}$-algebra-valued metric space $(X, \mathbb{A}, d)$ such that $d\left(x_{n+1}, x_{n}\right) \preceq P^{*} d\left(x_{n}, x_{n-1}\right) P, P \in \mathbb{A},\|P\|<1$. Then $\left\{x_{n}\right\}$ is a Cauchy sequence.

Proof. Let us consider $\lambda_{n}=\left\|d\left(x_{n+1}, x_{n}\right)\right\|$ and $0 \leq h=\|P\|^{2}<1$.

Therefore

$$
d\left(x_{n+1}, x_{n}\right) \preceq P^{*} d\left(x_{n}, x_{n-1}\right) P,
$$

which implies

$$
\lambda_{n}=\left\|d\left(x_{n+1}, x_{n}\right)\right\| \leq\left\|P^{*} d\left(x_{n}, x_{n-1}\right) P\right\|
$$




$$
\begin{aligned}
& \leq\left\|P^{*}\right\|\left\|d\left(x_{n}, x_{n-1}\right)\right\|\|P\| \\
& =\|P\|^{2}\left\|d\left(x_{n}, x_{n-1}\right)\right\| \\
& =h \lambda_{n-1} \text { for all } n \in \mathbb{N} .
\end{aligned}
$$

Then by Lemma 3.1, $\left\{x_{n}\right\}$ is a Cauchy sequence in $X$ with respect to $\mathbb{A}$.

Definition 3.3. Let $X$ be a non-empty set and $S, T: X \rightarrow X$. If $z=S x=T x$, for some $x \in X$, then $x$ is called a coincidence point of $S$ and $T$, and $z$ is called a point of coincidence of $S$ and $T$. If $z=x$, then $x$ is a common fixed point of $S$ and $T$.

Theorem 3.4. [7] If $(X, \mathbb{A}, d)$ is a complete $C^{*}$-algebra-valued metric space and $T$ is a contractive mapping, then there exists a fixed point of $T$ in $X$.

Theorem 3.5. Let $S$ and $T$ be two self-maps defined on a complete $C^{*}$-algebra-valued metric space $(X, \mathbb{A}, d)$, which satisfy the condition

$$
d(T x, S y) \preceq P^{*} M(x, y) P \quad \text { for any } x, y \in X,
$$

where $P \in \mathbb{A}$ with $\|P\|<\frac{1}{\sqrt{2}}$ and $M(x, y)$ takes either $d(x, y)$ or $d(T x, x)+$ $d(S y, y)$ or $d(T x, y)+d(S y, x)$.

Then $S$ and $T$ have a unique common fixed point in $X$.

Proof. Let $x_{0} \in X$. We define a sequence $\left\{x_{n}\right\}$ in $X$ by $x_{2 n+1}=S x_{2 n}, x_{2 n}=$ $T x_{2 n-1}$ for $n=1,2, \cdots$. Now we give the proof in the followING three cases:

Case 1 :

If $M(x, y)=d(x, y)$ for any $x, y \in X$. Then for $x \in X$, we have

$$
d(T x, S x) \preceq P^{*} M(x, x) P \Rightarrow d(T x, S x) \preceq P^{*} d(x, x) P=\theta .
$$

Therefore $d(S x, T x)=\theta$. Thus $S=T$. Hence the theorem is now same to Theorem 3.4.

Case 2 :

If $M(x, y)=d(T x, x)+d(S y, y)$ for any $x, y \in X$.

Now,

$$
\begin{aligned}
d\left(x_{2 n+2}, x_{2 n+1}\right) & =d\left(T x_{2 n+1}, S x_{2 n}\right) \\
& \preceq P^{*} M\left(x_{2 n+1}, x_{2 n}\right) P .
\end{aligned}
$$

Next,

$$
M\left(x_{2 n+1}, x_{2 n}\right)=d\left(T x_{2 n+1}, x_{2 n+1}\right)+d\left(S x_{2 n}, x_{2 n}\right)
$$




$$
=d\left(x_{2 n+2}, x_{2 n+1}\right)+d\left(x_{2 n+1}, x_{2 n}\right) .
$$

Therefore,

$$
\begin{aligned}
d\left(x_{2 n+2}, x_{2 n+1}\right) & \preceq P^{*}\left(d\left(x_{2 n+2}, x_{2 n+1}\right)+d\left(x_{2 n+1}, x_{2 n}\right)\right) P \\
& =P^{*} d\left(x_{2 n+2}, x_{2 n+1}\right) P+P^{*} d\left(x_{2 n+1}, x_{2 n}\right) P .
\end{aligned}
$$

Hence,

$$
\begin{aligned}
\left\|d\left(x_{2 n+2}, x_{2 n+1}\right)\right\| & \leq\left\|P^{*} d\left(x_{2 n+2}, x_{2 n+1}\right) P+P^{*} d\left(x_{2 n+1}, x_{2 n}\right) P\right\| \\
& \leq\left\|P^{*} d\left(x_{2 n+2}, x_{2 n+1}\right) P\right\|+\left\|P^{*} d\left(x_{2 n+1}, x_{2 n}\right) P\right\| \\
& \leq\|P\|^{2}\left\|d\left(x_{2 n+2}, x_{2 n+1}\right)\right\|+\|P\|^{2}\left\|d\left(x_{2 n+1}, x_{2 n}\right)\right\| .
\end{aligned}
$$

This implies,

$$
\left\|d\left(x_{2 n+2}, x_{2 n+1}\right)\right\| \leq \frac{\|P\|^{2}}{1-\|P\|^{2}}\left\|d\left(x_{2 n+1}, x_{2 n}\right)\right\| .
$$

Let $h=\frac{\|P\|^{2}}{1-\|P\|^{2}}$. Then $0 \leq h<1$. Hence,

$$
\left\|d\left(x_{2 n+2}, x_{2 n+1}\right)\right\| \leq h\left\|d\left(x_{2 n+1}, x_{2 n}\right)\right\| .
$$

Again,

$$
\begin{aligned}
d\left(x_{2 n+1}, x_{2 n}\right) & =d\left(S x_{2 n}, T x_{2 n-1}\right)=d\left(T x_{2 n-1}, S x_{2 n}\right) \\
& \preceq P^{*} M\left(x_{2 n-1}, x_{2 n}\right) P .
\end{aligned}
$$

Now,

$$
\begin{aligned}
M\left(x_{2 n-1}, x_{2 n}\right) & =d\left(T x_{2 n-1}, x_{2 n-1}\right)+d\left(S x_{2 n}, x_{2 n}\right) \\
& =d\left(x_{2 n}, x_{2 n-1}\right)+d\left(x_{2 n+1}, x_{2 n}\right) .
\end{aligned}
$$

Therefore,

$$
\begin{aligned}
d\left(x_{2 n+1}, x_{2 n}\right) & \preceq P^{*}\left(d\left(x_{2 n+1}, x_{2 n}\right)+d\left(x_{2 n}, x_{2 n-1}\right)\right) P \\
& =P^{*} d\left(x_{2 n+1}, x_{2 n}\right) P+P^{*} d\left(x_{2 n}, x_{2 n-1}\right) P .
\end{aligned}
$$

Hence,

$$
\begin{aligned}
\left\|d\left(x_{2 n+1}, x_{2 n}\right)\right\| & \leq\left\|P^{*} d\left(x_{2 n+1}, x_{2 n}\right) P+P^{*} d\left(x_{2 n}, x_{2 n-1}\right) P\right\| \\
& \leq\left\|P^{*} d\left(x_{2 n+1}, x_{2 n}\right) P\right\|+\left\|P^{*} d\left(x_{2 n}, x_{2 n-1}\right) P\right\|
\end{aligned}
$$




$$
\leq\|P\|^{2}\left\|d\left(x_{2 n+1}, x_{2 n}\right)\right\|+\|P\|^{2}\left\|d\left(x_{2 n}, x_{2 n-1}\right)\right\|
$$

This implies,

$$
\left\|d\left(x_{2 n+1}, x_{2 n}\right)\right\| \leq \frac{\|P\|^{2}}{1-\|P\|^{2}}\left\|d\left(x_{2 n}, x_{2 n-1}\right)\right\| .
$$

Hence,

$$
\left\|d\left(x_{2 n+1}, x_{2 n}\right)\right\| \leq h\left\|d\left(x_{2 n}, x_{2 n-1}\right)\right\| .
$$

Therefore, from (3) and (6), we have

$$
\left\|d\left(x_{n+1}, x_{n}\right)\right\| \leq h\left\|d\left(x_{n}, x_{n-1}\right)\right\| \text { for all } n \in \mathbb{N} .
$$

By Lemma 3.1, $\left\{x_{n}\right\}$ is a Cauchy sequence in $X$ with respect to $\mathbb{A}$. By the completeness of $(X, \mathbb{A}, d)$, there exists an $x \in X$ such that $\lim _{n \rightarrow \infty} x_{n}=x$. Now for $n \rightarrow \infty$,

$$
\begin{aligned}
\theta & \preceq d(x, T x) \preceq d\left(x_{2 n+1}, T x\right)=d\left(T x, S x_{2 n}\right) \\
& \preceq P^{*} M\left(x, x_{2 n}\right) P .
\end{aligned}
$$

Now as $n \rightarrow \infty$, we get

$$
\begin{aligned}
M\left(x, x_{2 n}\right) & =d(T x, x)+d\left(S x_{2 n}, x_{2 n}\right) \\
& =d(T x, x)+d\left(x_{2 n+1}, x_{2 n}\right) \\
& =d(T x, x) .
\end{aligned}
$$

Therefore,

$$
\theta \preceq d(x, T x) \preceq P^{*} d(x, T x) P .
$$

This implies,

$$
0 \leq\|d(x, T x)\| \leq\left\|P^{*} P\right\|\|d(x, T x)\|,
$$

a contradiction. Hence $T x=x$, i.e., $x$ is a fixed point of $T$. Now we show that $x$ is a fixed point of $S$.

$$
\theta \preceq d(x, S x)=d(T x, S x) \preceq P^{*} M(x, x) P=P^{*} d(S x, x) P .
$$

Since,

$$
M(x, x)=d(T x, x)+d(S x, x)=d(S x, x) .
$$


Therefore,

$$
\theta \preceq d(x, S x) \preceq P^{*} d(x, S x) P .
$$

This implies,

$$
0 \leq\|d(x, S x)\| \leq\left\|P^{*} P\right\|\|d(x, S x)\|,
$$

a contradiction. Thus $S x=x$, i.e., $x$ is a fixed point of $S$. Hence $x$ is a common fixed point of $S$ and $T$.

Finally, we show that $x$ is the unique common fixed point. Let $y$ be another common fixed point of $S$ and $T$. Then $y=S y=T y$. Now,

$$
\begin{aligned}
\theta & \preceq d(x, y)=d(T x, S y) \\
& \preceq P^{*} M(x, y) P,
\end{aligned}
$$

and

$$
M(x, y)=d(T x, x)+d(S y, y)=\theta
$$

Hence,

$$
\theta \preceq d(x, y) \preceq \theta
$$

This shows that $d(x, y)=\theta$. Hence $x=y$ and this implies that the common fixed point of $S$ and $T$ is unique.

Case 3 :

If $M(x, y)=d(T x, y)+d(S y, x)$ for any $x, y \in X$. Now,

$$
\begin{aligned}
d\left(x_{2 n+2}, x_{2 n+1}\right) & =d\left(T x_{2 n+1}, S x_{2 n}\right) \\
& \preceq P^{*} M\left(x_{2 n+1}, x_{2 n}\right) P .
\end{aligned}
$$

Next,

$$
\begin{aligned}
M\left(x_{2 n+1}, x_{2 n}\right) & =d\left(T x_{2 n+1}, x_{2 n}\right)+d\left(S x_{2 n}, x_{2 n+1}\right) \\
& =d\left(x_{2 n+2}, x_{2 n}\right)+d\left(x_{2 n+1}, x_{2 n+1}\right) \\
& =d\left(x_{2 n+2}, x_{2 n}\right) \\
& \preceq d\left(x_{2 n+2}, x_{2 n+1}\right)+d\left(x_{2 n+1}, x_{2 n}\right) .
\end{aligned}
$$

Therefore, by (1), (2) we get (3), i.e., we have

$$
\left\|d\left(x_{2 n+2}, x_{2 n+1}\right)\right\| \leq h\left\|d\left(x_{2 n+1}, x_{2 n}\right)\right\| .
$$


Again,

$$
\begin{aligned}
d\left(x_{2 n+1}, x_{2 n}\right) & =d\left(S x_{2 n}, T x_{2 n-1}\right)=d\left(T x_{2 n-1}, S x_{2 n}\right) \\
& \preceq P^{*} M\left(x_{2 n-1}, x_{2 n}\right) P .
\end{aligned}
$$

Now,

$$
\begin{aligned}
M\left(x_{2 n-1}, x_{2 n}\right) & =d\left(T x_{2 n-1}, x_{2 n}\right)+d\left(S x_{2 n}, x_{2 n-1}\right) \\
& =d\left(x_{2 n}, x_{2 n}\right)+d\left(x_{2 n+1}, x_{2 n-1}\right) \\
& =d\left(x_{2 n+1}, x_{2 n-1}\right) \\
& \preceq d\left(x_{2 n+1}, x_{2 n}\right)+d\left(x_{2 n}, x_{2 n-1}\right) .
\end{aligned}
$$

Therefore, by (4), (5) we get (6), i.e., we have

$$
\left\|d\left(x_{2 n+1}, x_{2 n}\right)\right\| \leq h\left\|d\left(x_{2 n}, x_{2 n-1}\right)\right\| .
$$

Therefore, from (3) and (6) we get (7), i.e.,

$$
\left\|d\left(x_{n+1}, x_{n}\right)\right\| \leq h\left\|d\left(x_{n}, x_{n-1}\right)\right\|, \text { for all } n \in \mathbb{N} .
$$

Again by Lemma 3.1, $\left\{x_{n}\right\}$ is a Cauchy sequence in $X$ with respect to $\mathbb{A}$. By the completeness of $(X, \mathbb{A}, d)$, there exists an $x \in X$ such that $\lim _{n \rightarrow \infty} x_{n}=x$. Now, for $n \rightarrow \infty$, we have

$$
\begin{aligned}
\theta & \preceq d(x, T x) \preceq d\left(x_{2 n+1}, T x\right)=d\left(T x, S x_{2 n}\right) \\
& \preceq P^{*} M\left(x, x_{2 n}\right) P .
\end{aligned}
$$

Now, as $n \rightarrow \infty$

$$
\begin{aligned}
M\left(x, x_{2 n}\right) & =d\left(T x, x_{2 n}\right)+d\left(S x_{2 n}, x\right) \\
& =d\left(T x, x_{2 n}\right)+d\left(x_{2 n+1}, x\right) \\
& =d(T x, x) .
\end{aligned}
$$

Therefore,

$$
\theta \preceq d(x, T x) \preceq P^{*} d(x, T x) P,
$$

this implies,

$$
0 \leq\|d(x, T x)\| \leq\left\|P^{*} P\right\|\|d(x, T x)\|,
$$

a contradiction. Hence $T x=x$, i.e., $x$ is a fixed point of $T$. 
Again, we show that, $x$ is a fixed point of $S$.

$$
\theta \preceq d(x, S x)=d(T x, S x) \preceq P^{*} M(x, x) P=P^{*} d(S x, x) P,
$$

since,

$$
M(x, x)=d(T x, x)+d(S x, x)=d(S x, x) .
$$

Therefore,

$$
\theta \preceq d(x, S x) \preceq P^{*} d(x, S x) P,
$$

this implies,

$$
0 \leq\|d(x, S x)\| \leq\left\|P^{*} P\right\|\|d(x, S x)\|
$$

a contradiction. Thus $S x=x$, i.e., $x$ is a fixed point of $S$. Hence $x$ is a common fixed point of $S$ and $T$.

Finally, we show that common fixed point is unique. Let $y$ be another common fixed point of $S$ and $T$. Then $y=S y=T y$. Now,

$$
\begin{aligned}
\theta & \preceq d(x, y)=d(T x, S y) \\
& \preceq P^{*} M(x, y) P .
\end{aligned}
$$

Therefore,

$$
\begin{aligned}
M(x, y)=d(T x, y)+d(S y, x) \preceq d(T x, x)+d(x, y)+d(S y, y)+ & d(y, x) \\
& =2 d(x, y)
\end{aligned}
$$

or

$$
\theta \preceq d(x, y) \preceq P^{*} 2 d(x, y) P .
$$

This implies,

$$
0 \leq\|d(x, y)\| \leq 2\left\|P^{*} P\right\|\|d(x, y)\|<\|d(x, y)\| .
$$

This shows that $d(x, y)=\theta$. Hence $x=y$ and this implies that the common fixed point of $S$ and $T$ is unique.

If one goes through the proof of Theorem 3.5, one can easily verify the following result. 
Corollary 3.6. Let $(X, \mathbb{A}, d)$ be a complete $C^{*}$-algebra-valued metric space. Suppose that the mappings $T, S: X \rightarrow X$ satisfy

$$
\begin{aligned}
& \|d(S x, T y)\| \\
& \quad \leq\|P\| \max \{\|d(x, y)\|,\|d(T x, x)+d(S y, y)\|,\|d(T x, y)+d(S y, x)\|\},
\end{aligned}
$$

for any $x, y \in X$, where $P \in \mathbb{A},\|P\|<\frac{1}{\sqrt{2}}$. Then $T$ and $S$ have a unique common fixed point in $X$.

Definition 3.7. (cf. [7]) Let $X$ be a non-empty set. We call a mapping $T$ is a $C^{*}$-algebra-valued expansion mapping on $X$, if $T: X \rightarrow X$ satisfies:

(1) $T(X)=X$

(2) $d(T x, T y) \succeq P^{*} d(x, y) P$ for all $x, y \in X$,

where $P \in \mathbb{A}$, is an invertible element and $\left\|P^{-1}\right\|<1$.

Theorem 3.8. Let $S$ and $T$ be two expansion mappings on a complete $C^{*}$-algebra-valued metric space $(X, \mathbb{A}, d)$ such that for all $x, y \in X$

$$
d(S x, T y) \succeq P^{*} d(x, y) P,
$$

where $P \in \mathbb{A}$, is an invertible element and $\left\|P^{-1}\right\|<1$. Then $S$ and $T$ have a unique common fixed point.

Proof. Firstly, we claim that $S$ is injective. If $S$ is not injective, then there are some $x, y \in X$, with $x \neq y$, such that $S x=S y$. As $S$ is an expansion mapping, we have

$$
\theta=d(S x, S y) \succeq P^{*} d(x, y) P .
$$

Since $P^{*} d(x, y) P \in \mathbb{A}_{+}, P^{*} d(x, y) P=\theta$. Also $P$ is invertible, so $d(x, y)=\theta$, which is impossible. Thus $S$ is injective.

In similar argument, $T$ is also injective.

Now for all $x, y \in X$

$$
d(S x, T y) \succeq P^{*} d(x, y) P .
$$

In the above formula, substituting $x, y$ with $S^{-1} x, T^{-1} y$ respectively, we get

$$
d(x, y) \succeq P^{*} d\left(S^{-1} x, T^{-1} y\right) P .
$$

This implies

$$
\left(P^{*}\right)^{-1} d(x, y) P^{-1} \succeq d\left(S^{-1} x, T^{-1} y\right)
$$


Hence

$$
\left(P^{-1}\right)^{*} d(x, y) P^{-1} \succeq d\left(S^{-1} x, T^{-1} y\right) .
$$

Therefore by Theorem 3.5, there exists a unique $x \in X$ such that $x$ is a common fixed point of $S^{-1}$ and $T^{-1}$.

Thus

$$
S^{-1} x=x=T^{-1} x .
$$

This implies that there exists a unique $x \in X$ such that

$$
S x=x=T x .
$$

Before going to another theorem, we recall the following Lemma of [5].

Lemma 3.9. Suppose that $\mathbb{A}$ is a unital $C^{*}$-algebra with a unit $I$.

(1) If $a \in \mathbb{A}_{+}$with $\|a\|<\frac{1}{2}$, then $I-a$ is invertible and $\|a(I-a)\|<1$;

(2) suppose $a, b \in \mathbb{A}$ with $a, b \succeq \theta$ and $a b=b a$, then $a b \succeq \theta$;

(3) by $\mathbb{A}^{\prime}$, we denote the set $\{a \in \mathbb{A}: a b=b a, \forall b \in \mathbb{A}\}$. Let $a \in \mathbb{A}^{\prime}$, if $b, c \in \mathbb{A}$ with $b \succeq c \succeq \theta$ and $I-a \in \mathbb{A}_{+}^{\prime}$ is an invertible operator, then

$$
(I-a)^{-1} b \succeq(I-a)^{-1} c .
$$

Notice that in a $C^{*}$-algebra, $\theta \preceq a, b$, does not imply $\theta \preceq a b$, see [7].

Theorem 3.10. Let $(X, \mathbb{A}, d)$ be a complete $C^{*}$-algebra-valued metric space. Suppose the self-mappings $S, T$ on $X$ satisfy

$$
d(S x, T y) \preceq P(d(S x, y)+d(T y, x)),
$$

for all $x, y \in X$, where $P \in \mathbb{A}_{+}^{\prime}$ and $\|P\|<\frac{1}{2}$. Then $S$ and $T$ have a unique common fixed point.

Proof. Suppose $P \neq \theta$. As $P \in \mathbb{A}_{+}^{\prime}, P(d(S x, y)+d(T y, x))$ is also a positive element. Let $x_{0} \in X$. We define a sequence $\left\{x_{n}\right\}$ in $X$ by $x_{2 n+1}=S x_{2 n}, x_{2 n}=$ $T x_{2 n-1}$ for $n=1,2, \cdots$.

Now

$$
\begin{aligned}
d\left(x_{2 n+1}, x_{2 n+2}\right) & =d\left(S x_{2 n}, T x_{2 n+1}\right) \\
& \preceq P\left(d\left(S x_{2 n}, x_{2 n+1}\right)+d\left(T x_{2 n+1}, x_{2 n}\right)\right)
\end{aligned}
$$




$$
\begin{aligned}
& =P\left(d\left(S x_{2 n}, S x_{2 n}\right)+d\left(T x_{2 n+1}, T x_{2 n-1}\right)\right) \\
& \preceq P\left(d\left(T x_{2 n+1}, S x_{2 n}\right)+d\left(S x_{2 n}, T x_{2 n-1}\right)\right) \\
& =P\left(d\left(x_{2 n+2}, x_{2 n+1}\right)+d\left(x_{2 n+1}, x_{2 n}\right)\right) \\
& =P d\left(x_{2 n+2}, x_{2 n+1}\right)+P d\left(x_{2 n+1}, x_{2 n}\right) .
\end{aligned}
$$

Thus $(I-P) d\left(x_{2 n+1}, x_{2 n+2}\right) \preceq P d\left(x_{2 n}, x_{2 n+1}\right)$.

Again

$$
\begin{aligned}
d\left(x_{2 n+1}, x_{2 n}\right) & =d\left(S x_{2 n}, T x_{2 n-1}\right) \\
& \preceq P\left(d\left(S x_{2 n}, x_{2 n-1}\right)+d\left(T x_{2 n-1}, x_{2 n}\right)\right) \\
& =P\left(d\left(S x_{2 n}, S x_{2 n-2}\right)+d\left(T x_{2 n-1}, T x_{2 n-1}\right)\right) \\
& \preceq P\left(d\left(S x_{2 n}, T x_{2 n-1}\right)+d\left(T x_{2 n-1}, S x_{2 n-2}\right)\right) \\
& =P\left(d\left(x_{2 n+1}, x_{2 n}\right)+d\left(x_{2 n}, x_{2 n-1}\right)\right) \\
& =P d\left(x_{2 n+1}, x_{2 n}\right)+P d\left(x_{2 n}, x_{2 n-1}\right) .
\end{aligned}
$$

Thus

$$
(I-P) d\left(x_{2 n+1}, x_{2 n}\right) \preceq P d\left(x_{2 n}, x_{2 n-1}\right) .
$$

Therefore

$$
(I-P) d\left(x_{n+1}, x_{n}\right) \preceq P d\left(x_{n}, x_{n-1}\right) .
$$

Since $P \in \mathbb{A}_{+}^{\prime}$ with $\|P\|<\frac{1}{2}$, we have $(I-P)^{-1} \in \mathbb{A}_{+}^{\prime}$. By Lemma 3.9

$$
P(I-P)^{-1} \in \mathbb{A}_{+}^{\prime} \text { with }\left\|P(I-P)^{-1}\right\|<1 .
$$

Therefore

$$
(I-P) d\left(x_{n+1}, x_{n}\right) \preceq P d\left(x_{n}, x_{n-1}\right),
$$

which gives

$$
d\left(x_{n+1}, x_{n}\right) \preceq P(I-P)^{-1} d\left(x_{n}, x_{n-1}\right) .
$$

We consider $\left\|P(I-P)^{-1}\right\|=h$ and $\lambda_{n}=\left\|d\left(x_{n+1}, x_{n}\right)\right\|$. Then

$$
d\left(x_{n+1}, x_{n}\right) \preceq P(I-P)^{-1} d\left(x_{n}, x_{n-1}\right) .
$$

Thus

$$
\begin{aligned}
\lambda_{n}=\left\|d\left(x_{n+1}, x_{n}\right)\right\| & \leq\left\|P(I-P)^{-1} d\left(x_{n}, x_{n-1}\right)\right\| \\
& =\left\|P(I-P)^{-1}\right\|\left\|d\left(x_{n}, x_{n-1}\right)\right\| \\
& =h\left\|d\left(x_{n}, x_{n-1}\right)\right\| \\
& =h \lambda_{n-1} \text { with } h \in[0,1) .
\end{aligned}
$$


By Lemma 3.1, $\left\{x_{n}\right\}$ is a Cauchy sequence in $X$ with respect to $\mathbb{A}$. By the completeness of $(X, \mathbb{A}, d)$, there exists $x \in X$ such that $\lim _{n \rightarrow \infty} x_{n}=x$.

Since

$$
\begin{aligned}
d(S x, x) & =d\left(S x, T x_{2 n+1}\right) \\
& \preceq d\left(S x, T x_{2 n+1}\right)+d\left(T x_{2 n+1}, x\right) \\
& \preceq P\left(d\left(S x, x_{2 n+1}\right)+d\left(T x_{2 n+1}, x\right)\right)+d\left(x_{2 n+2}, x\right) \\
&
\end{aligned}
$$

This means that

$$
(I-P) d(S x, x) \preceq P\left(d\left(x, x_{2 n+1}\right)+d\left(x_{2 n+2}, x\right)\right)+d\left(x_{2 n+2}, x\right) .
$$

Therefore

$$
\begin{aligned}
\|d(S x, x)\| \leq & \left\|P(I-P)^{-1}\right\|\left\|\mid\left(d\left(x, x_{2 n+1}\right)+d\left(x_{2 n+2}, x\right)\right)\right\| \\
& +\left\|(I-P)^{-1}\right\|\left\|d\left(x_{2 n+2}, x\right)\right\| \\
\leq & \left\|P(I-P)^{-1}\right\|\left(\left\|d\left(x, x_{2 n+1}\right)\right\|+\left\|d\left(x_{2 n+2}, x\right)\right\|\right) \\
& +\left\|(I-P)^{-1}\right\|\left\|d\left(x_{2 n+2}, x\right)\right\| \\
\rightarrow & 0 \text { as } n \rightarrow \infty .
\end{aligned}
$$

Hence $S x=x$, i.e., $x$ is a fixed point of $S$. Similarly, it is easy to show that $x$ is a fixed point of $T$. Therefore $x$ is common fixed point of $S$ and $T$.

To prove the uniqueness, we consider $y(\neq x)$ as another common fixed point of $S$ and $T$.

Therefore

$$
d(S y, y)=y=d(T y, y) .
$$

Now we have,

$$
\begin{aligned}
\theta & \preceq d(x, y)=d(S x, T y) \\
& \preceq P(d(S x, y)+d(T y, x)) \\
& =P(d(x, y)+d(y, x))
\end{aligned}
$$

which implies

$$
d(x, y) \preceq P(I-P)^{-1} d(x, y) .
$$

Therefore since $\left\|P(I-P)^{-1}\right\|<1$,

$$
0 \leq\|d(x, y)\|=\|d(S x, T y)\|
$$




$$
\begin{aligned}
& \leq\left\|P(I-P)^{-1} d(x, y)\right\| \\
& \leq\left\|P(I-P)^{-1}\right\|\|d(x, y)\| \\
& <\|d(x, y)\| .
\end{aligned}
$$

Hence $d(x, y)=\theta \Leftrightarrow x=y$. This completes the proof.

Remark 3.11. If we consider $S=T$, then the Theorems $2.1-2.3$ of [7] are the special cases of the above results.

\section{Best Proximity Point Result}

In this section, we define $C^{*}$-algebra-valued proximal contractions and set up the way for the best proximity point theorem on $C^{*}$-algebra-valued metric spaces. For this, we give the following definitions and notations.

Definition 4.1. Let $(X, \mathbb{A}, d)$ be a $C^{*}$-algebra-valued metric space and $A$ and $B$ be two non-empty subsets of $X$. Let $T: A \rightarrow B$ be a mapping. A point $x \in A$ is said to be a best proximity point of $T$, if it satisfies the condition that

$$
d(x, T x)=d(A, B),
$$

where $d(A, B)=\inf \{d(x, y) \in \mathbb{A}: x \in A, y \in B$ and $d(x, y)=\|x-y\| I\}$.

If $B=A$, and $A$ is complete, then the best proximity point reduces to the normal fixed point of $T$.

Let $A$ and $B$ be two non-empty subsets of a $C^{*}$-algebra-valued metric space $(X, \mathbb{A}, d)$. We define $A_{0}$ and $B_{0}$ as,

$$
A_{0}=\{x \in A: d(x, y)=d(A, B) \text { for some } y \in B\}
$$

and

$$
B_{0}=\{y \in B: d(x, y)=d(A, B) \text { for some } x \in A\} .
$$

Definition 4.2. [4] Let $(A, B)$ be a pair of non-empty subsets of a complete metric space $(X, d)$. A mapping $T: A \rightarrow B$ is said to be a proximal contraction if there exists a non-negative real number $\alpha<1$ such that, for all $u_{1}, u_{2}, x_{1}, x_{2} \in$ A,

$$
\left.\begin{array}{l}
d\left(u_{1}, T x_{1}\right)=d(A, B) \\
d\left(u_{2}, T x_{2}\right)=d(A, B
\end{array}\right\} \Rightarrow d\left(u_{1}, u_{2}\right) \leq \alpha d\left(x_{1}, x_{2}\right)
$$


Definition 4.3. Let $(A, B)$ be a pair of non-empty subsets of a complete $C^{*}$-algebra-valued metric space $(X, \mathbb{A}, d)$. A mapping $T: A \rightarrow B$ is said to be a $C^{*}$-algebra-valued proximal contraction if there exists a $P \in \mathbb{A}$ with $\|P\|<1$ such that, for all $u_{1}, u_{2}, x_{1}, x_{2} \in A$,

$$
\left.\begin{array}{l}
d\left(u_{1}, T x_{1}\right)=d(A, B) \\
d\left(u_{2}, T x_{2}\right)=d(A, B
\end{array}\right\} \Rightarrow d\left(u_{1}, u_{2}\right) \preceq P^{*} d\left(x_{1}, x_{2}\right) P .
$$

Now, we are in a position to give the following result.

Theorem 4.4. Let $(A, B)$ be a pair of non-empty closed subsets of a complete $C^{*}$-algebra-valued metric space $(X, \mathbb{A}, d)$ and $A_{0}$ be non-empty. We assume that $T: A \rightarrow B$ satisfies the following conditions:

(a) $T$ is a $C^{*}$-algebra-valued proximal contraction;

(b) $T\left(A_{0}\right) \subseteq B_{0}$.

Then there exists a unique $x \in A_{0}$ such that

$$
d(x, T x)=d(A, B) .
$$

Proof. Since $A_{0}$ is non-empty and $T\left(A_{0}\right) \subseteq B_{0}$, we choose $x_{0} \in A_{0}$. Then there exists $x_{1} \in A_{0}$ such that $d\left(x_{1}, T x_{0}\right)=d(A, B)$. Since $T x_{1} \in B_{0}$, there exists $x_{2} \in A_{0}$ such that $d\left(x_{2}, T x_{1}\right)=d(A, B)$. Continuing this process, we obtain a sequence $\left\{x_{n}\right\} \subseteq A_{0}$ such that

$$
d\left(x_{n+1}, T x_{n}\right)=d(A, B), \text { for all } n \in \mathbb{N} .
$$

As $T$ is a $C^{*}$-algebra-valued proximal contraction, there exists $P \in \mathbb{A}$ with $\|P\|<1$, for all $n \in \mathbb{N}$, such that

$$
\begin{aligned}
& d\left(x_{n}, T x_{n-1}\right)=d(A, B), \\
& d\left(x_{n+1}, T x_{n}\right)=d(A, B),
\end{aligned}
$$

we have

$$
d\left(x_{n+1}, x_{n}\right) \preceq P^{*} d\left(x_{n}, x_{n-1}\right) P .
$$

Then by Lemma 3.2, $\left\{x_{n}\right\}$ is a Cauchy sequence in $X$ with respect to $\mathbb{A}$. By the completeness of $(X, \mathbb{A}, d)$, there exists $x \in A$ such that $\lim _{n \rightarrow \infty} x_{n}=x$, since $A$ is closed. As $T\left(A_{0}\right) \subseteq B_{0}$, there exists an element $u \in A_{0}$ such that

$$
d(u, T x)=d(A, B)
$$


Also we have for all $n \in \mathbb{N}$,

$$
d\left(x_{n+1}, T x_{n}\right)=d(A, B) .
$$

Since $T$ is a $C^{*}$-algebra-valued proximal contraction, for all $n \in \mathbb{N}$,

$$
d\left(u, x_{n+1}\right) \preceq P^{*} d\left(x, x_{n}\right) P \rightarrow \theta \text { as } n \rightarrow \infty .
$$

Therefore $x_{n} \rightarrow u$ and $x=u$.

Thus

$$
d(x, T x)=d(A, B) .
$$

Now we shall prove the uniqueness of the best proximity point of $T$. Let us suppose $x, y$, with $x \neq y$, be two best proximity points of $T$ in $A$.

Therefore

$$
d(x, T x)=d(A, B) \text { and } d(y, T y)=d(A, B)
$$

Since $T$ is a $C^{*}$-algebra-valued proximal contraction

$$
\theta \preceq d(x, y) \preceq P^{*} d(x, y) P,
$$

we have

$$
\begin{aligned}
0 \leq\|d(x, y)\| & \leq\left\|P^{*} d(x, y) P\right\| \\
& \leq\left\|P^{*}\right\|\|d(x, y)\|\|P\| \\
& \leq\|P\|^{2}\|d(x, y)\| \\
& <\|d(x, y)\|,
\end{aligned}
$$

which is a contradiction. So $d(x, y)=\theta$ and $x=y$.

Therefore the best proximity point is unique.

\section{Application}

Fixed point theorems and common fixed point theorems for operators in ordered metric spaces are widely investigated and have been found various applications in differential equations and integral equations (see [6, 7, 9] and references therein). The common fixed point theorems proved here pave the way for an application on complete $C^{*}$-algebra-valued metric spaces to prove the existence and uniqueness of the solutions of the following integral equations. 
Theorem 5.1. Consider the integral equations

$$
\begin{aligned}
& x(t)=\int_{E} K_{1}(t, s, x(s)) d s+g(t), \quad t \in E, \\
& x(t)=\int_{E} K_{2}(t, s, x(s)) d s+g(t), \quad t \in E,
\end{aligned}
$$

where $E$ is a Lebesgue measurable set with finite measure.

Suppose that

(1) $K_{1}, K_{2}: E \times E \times \mathbb{R} \rightarrow \mathbb{R}$ and $g: E \rightarrow \mathbb{R}$ are members of $L^{\infty}(E)$.

(2) there exists a continuous function $\psi: E \times E \rightarrow \mathbb{R}$ and $k \in\left(0, \frac{1}{\sqrt{2}}\right)$ such that for $t, s \in E$ and $u, v \in \mathbb{R}$,

$$
\left|K_{1}(t, s, u)-K_{2}(t, s, v)\right| \leq k|\psi(t, s)(u-v)|,
$$

(3) $\sup _{t \in E} \int_{E}|\psi(t, s)| d s \leq 1$.

Then the integral equations (8) and (9) have a unique solution in $L^{\infty}(E)$.

Proof. Suppose $X=L^{\infty}(E)$ and $H=L^{2}(E)$.

We define $d: X \times X \rightarrow L(H)$ by

$$
d(f, g)=\pi_{|f-g|}(\forall f, g \in X),
$$

where $\pi_{h}: H \rightarrow H$ is the multiplication operator defined by

$$
\pi_{h}(\phi)=h . \phi,
$$

for $\phi \in H$. Then $d$ is a $C^{*}$-algebra-valued metric and $(X, L(H), d)$ is a complete $C^{*}$-algebra-valued metric space (see [7]).

Let $S, T: L^{\infty}(E) \rightarrow L^{\infty}(E)$ be

$$
\begin{aligned}
& S(x(t))=\int_{E} K_{1}(t, s, x(s)) d s+g(t), \quad t \in E, \\
& T(x(t))=\int_{E} K_{2}(t, s, x(s)) d s+g(t), \quad t \in E .
\end{aligned}
$$

Set $P=k I$. Then $P \in L(H)_{+}$and $\|P\|=k<\frac{1}{\sqrt{2}}$. Thus for any $h \in H$, we have,

$$
\|d(S x, T y)\|=\sup _{\|h\|=1}\left\|\pi_{|S x-T y|}(h)\right\|
$$




$$
\begin{aligned}
& =\sup _{\|h\|=1} \int_{E}\left[\left|\int_{E}\left(K_{1}(t, s, x(s))-K_{2}(t, s, y(s))\right) d s\right|\right] h(t) \overline{h(t)} d t \\
& \leq \sup _{\|h\|=1} \int_{E}\left[\int_{E}\left|K_{1}(t, s, x(s))-K_{2}(t, s, y(s))\right| d s\right]|h(t)|^{2} d t \\
& \leq \sup _{\|h\|=1} \int_{E}\left[\int_{E}|k \psi(t, s)(x(s)-y(s))| d s\right]|h(t)|^{2} d t \\
& \leq \quad k \sup _{\|h\|=1} \int_{E}\left[\int_{E}|\psi(t, s)| d s\right]|h(t)|^{2} d t .\|x-y\|_{\infty} \\
& \leq \quad k \sup _{t \in E} \int_{E}|\psi(t, s)| d s . \sup \int_{E}|h(t)|^{2} d t .\|x-y\|_{\infty} \\
& \leq \quad k\|x-y\|_{\infty} \quad\|h\|=1 \\
& =\|P\|\|d(x, y)\| \\
& =\|P\| \max \{\|d(x, y)\|,\|d(T x, x)+d(S y, y)\|, \\
&
\end{aligned}
$$

Since, it can be easily show that

$$
\max \{\|d(x, y)\|,\|d(T x, x)+d(S y, y)\|,\|d(T x, y)+d(S y, x)\|\}=\|d(x, y)\| .
$$

Since $\|P\|<\frac{1}{\sqrt{2}}$, then by Corollary 3.6, the integral equations (8) and (9) have a unique solution.

\section{Acknowledgments}

The second and third named author would like to convey their cordial thanks to DST-INSPIRE, New Delhi, India and CSIR, New Delhi, India respectively for their financial support. We gratefully acknowledge the critical review by the learned referees which improves the manuscript.

\section{References}

[1] S. Banach, Sur les opérations dans les ensembles abstraits et leurs application aux équations intégrales, Fund. Math., 3 (1922), 133-181.

[2] D.W. Boyd, J.S.W. Wong, On nonlinear contraction, Proc. Amer. Math. Soc., 20 (1969), 458-464.

[3] A. Chanda, S. Mondal, L.K. Dey, S. Karmakar, $C^{*}$-algebra-valued contractive mappings with its applications to integral equations, Indian J. Math., To Appear. 
[4] J. Chen, S. Xiao, H. Wang, and S.Deng, Best proximity point for the proximal nonexpansive mapping on the starshaped sets, Fixed Point Theory Appl., 19 (2015).

[5] R.G. Douglas, Banach Algebra Techniques in Operator Theory, Springer, Berlin, 1998.

[6] N. Hussain, M.H. Shah, A.A. Harandi, Z. Akhtar, Common fixed point theorems for generalized contractive mappings with applications, Fixed Point Theory Appl., 169 (2013).

[7] Z. Ma, L. Jiang, H. Sun, $C^{*}$-algebra-valued metric spaces and related fixed point theorems, Fixed Point Theory Appl., 206 (2014).

[8] G.J. Murphy, $C^{*}$-Algebras and Operator Theory, Academic Press, London, 1990.

[9] H.K. Pathak, M.S. Khan, R. Tiwari, A common fixed point theorem and its application to nonlinear integral equations, Comput. Math. Appl., 53, No. 2 (2007), 961-971.

[10] I. Segal, Irreducible representations of operator algebras, Bull. Amer. Math. Soc. (N.S.), 53, No. 2 (1947), 73-88.

[11] Q.H. Xu, T.E.D. Bieke, Z.Q. Chen, Introduction to Operator Algebras and Noncommutative $L^{\mathrm{p}}$ Spaces, Science Press, Beijing, 2010 (In Chinese). 